\title{
EL MODELO DE CRECIMIENTO ECONÓMICO EN ECUADOR EN EL PERÍODO 2004 - 2015: UNA PERSPECTIVA A PARTIR DE LA LEY DE THIRLWALL
}

\author{
THE MODEL OF ECONOMIC GROWTH IN ECUADOR FROM 2004 TO \\ 2015: A PERSPECTIVE BASED ON THE THIRLWALL LAW
}

\author{
Sebastián Cárdenas Z ${ }^{* 1}$.; Edison Vaca V. ${ }^{1}$; Martha Alvarado V. ${ }^{1}$ \\ 1Universidad de Especialidades Espíritu Santo UEES., Facultad de Economía y Ciencias Empresariales, \\ scardenasz@uees.edu.ec; edd_2024@hotmail.com; malvarve@uees.edu.ec
}

RESUMEN

ABSTRACT

El objetivo principal de este escrito fue analizar de manera descriptiva las restricciones de las variables de crecimiento del Ecuador del periodo 2004 al 2015 usando la literatura de Thirlwall; así como describir brevemente los principales hechos históricos que contribuyeron a cada etapa. Se empleó como metodología el análisis mediante histogramas con líneas de tendencia, lo cual fue contrastado teóricamente con el apartado de restricción de la balanza de pagos de la Ley de Thirlwall. Hallamos que, en la balanza de pagos de Ecuador existe un lento incremento en las tasas de exportaciones a diferencia de las importaciones, por lo cual, según Thirlwall, existe una restricción en la balanza de pagos que delimita el crecimiento del comercio exterior y es crucial para el desempeño económico, concluyendo que la falta de competitividad se ha hecho presente en el país.

PALABRAS CLAVES: balanza de pagos, crecimiento económico, comercio exterior, exportaciones, importaciones, tasa de crecimiento.

The main objective of this paper was to analyze descriptively the restrictions of Ecuador's growth variables from 2004 to 2015 using the Thirlwall literature; as well as briefly describe the main historical facts that contributed to each stage.

The descriptive analysis was used by histograms with trend lines was used as a methodology, which was theoretically contrasted with the restriction section of the balance of payments of the Thirlwall Law.

We found that, in Ecuador's balance of payments there is a slow increase in export rates as opposed to imports, which, according to Thirlwall, there is a balance of payments restriction that limits the growth of foreign trade and is crucial for economic performance, concluding that the lack of competitiveness has become present in the country.

KEY WORDS: balance of payments, economic growth, foreign trade, exports, imports, growth rate. 


\section{Introducción}

El crecimiento económico es un pilar clave dentro del desarrollo estructural de todo país según Ortiz (2013), que no se da de la noche a la mañana, sino que depende de un largo trayecto que contempla una serie de transformaciones por implementación de objetivos económicos a través de políticas planificadas por el ejecutivo (Rivera \& Toledo, 2004).

El crecimiento económico de un país se puede alimentar de fundamentalmente de dos sectores: el interno y el externo, de los cuales, el sector externo nos habla específicamente de todas las relaciones internacionales que tiene una economía, llámense actividades, intercambios o concordatos con miras a expansión o no (Ortiz, 2013).

Para un país dolarizado, este sector básicamente sustenta el tipo de cambio en nuestra economía por medio de las inversiones de bienes de capital, los vencimientos de deudas, exportaciones, etc. (Ortiz, 2013). Dentro de esta temática, la ley de Thirlwall, nos dice que las exportaciones tienen un papel significativo dentro del equilibrio a largo plazo en una economía, ya que parte de la mismas, financian el déficit en la cuenta corriente de la balanza de pagos (producto de tasas de crecimiento efectivas elevadas en comparación a las tasas de crecimiento consistente en el largo plazo) (Ortiz, 2013).

Según Bértola (2000) "si un país tiene un patrón de especialización productiva y un nivel de ingresos que determinan que la elasticidad ingreso de la demanda de sus exportaciones sea menor que sus contrapartes comerciales para mantener el equilibrio de la balanza comercial” (pág. 147).

Así, la extensión que proporciona el mercado mediante la demanda de bienes y servicios, es compatible con la expansión económica. El dinamismo que tiene la demanda externa (exportaciones) como componente de la demanda agregada, tiene relación directa en cuanto esta aumenta porque se contempla un escenario deseable para modelos de crecimiento/expansión en producción agregada (Ortiz, 2013).

Por otra parte, a lo largo de la historia del pensamiento económico, las escuelas clásicas y neoclásicas fundamentan que una nación tiene potencial de crecimiento solamente si esta acumula los recursos suficientes. De esta manera, los ciclos de crecimiento tenían mucha relación con el sector externo en los países más abiertos a innovar con nuevos agregados usando sus recursos locales en mayor proporción a aquellos que son importados.

El enfoque Harrod-Domar indica que variables como el capital y el trabajo no son consideradas como restricción al crecimiento porque simplemente no están orientadas a la demanda agregada. Sala-I-Martin (2000) declara lo siguiente "supongamos que el aumento del capital que se precisa para aumentar la producción en cantidad sea un valor constante. En particular, es un valor independiente de la relación capital-trabajo. Es decir, $\Delta \mathrm{Y}_{-} \mathrm{t}=\mathrm{A} \Delta \mathrm{K}_{-} \mathrm{t}^{\prime}$ siendo A una constante" (págs. 70-71).

Dicho modelo no contiene funciones de producción de forma explícita (como el costo de oportunidad que se genera), lo cual indica que para una versión simplificada de la función como la AK (de crecimiento endógeno), los factores de trabajo no juegan ningún papel importante en comparación al capital. Más bien los factores de capital y trabajo restringen a la oferta agregada debido a que limitan la dinámica de los recursos naturales basada en el aumento de la producción.

Según De Gregorio (2007) el modelo AK "intenta explicar la posibilidad de que el crecimiento se pueda sostener sin necesidad de suponer alguna fuerza externa" (pág. 315). Así, al surgir nuevas interrogantes como el porqué del crecimiento y a la vez el empobrecimiento de las economías mundiales, estos fenómenos hicieron que el modelo Harrod-Domar pierda credibilidad en el largo plazo, pues este modelo no explicaba el crecimiento económico sino más bien el crecimiento-inversión y la relación que tenía con el desempleo.

De esta manera, las demandas agregadas de todos los países no son estandarizadas, sino que difieren debido a que no existe equidad, ni mercados perfectos, ni tasas de crecimiento constantes.

Es decir, mientras más apertura tenga un país hacia el comercio, más peso tendrán las cifras sobre la balanza de pagos en relación a las tasas del producto interno en una población. Según Parkín (2004) "en el mundo moderno, también reconocemos el efecto de los cambios en las exportaciones sobre la demanda agregada" (pág. 699).

Thirlwall declaraba que en el largo plazo, el panorama para el crecimiento de un país es un hecho de que las exportaciones tengan un cumplimiento cíclico en el tiempo, ya que a medida que haya desarrollo la tasa de crecimiento de las exportaciones irá decreciendo a tal medida que las importaciones las superen, lo cual ocasiona que el equilibrio en la cuenta corriente de la balanza de pagos sufra inestabilidades, lo que incurre a que países con problemas constantemente se encuentre evaluando las posibilidades de financiamiento.

La solución que propone Thirlwall en el crecimiento económico, propone que todo modelo de crecimiento natural debería darse por la expansión en las exportaciones. El crecimiento de la demanda del comercio externo no genera déficits dentro de la cuenta corriente nacional, al mismo tiempo, estos indicadores limitan a que las economías no se endeuden de sobre manera. De acuerdo a las múltiples reflexiones so- 
bre el modelo de Thirlwall, Molina \& Zárate (2009) manifiestan que "la idea (...) es establecer que en una economía abierta, la demanda de exportaciones es el componente más importante (...) De tal manera que el crecimiento en las exportaciones dirigirá al crecimiento del producto en el largo plazo" (pág. 24).

Esta teoría sugestiona que si bien para el crecimiento que propone Thirlwall es necesario un aumento en la elasticidad de las exportaciones, paralelamente también se debe inferir para reducir las importaciones.

Finalmente, según la CEPAL (2006) "si la capacidad para importar no se expande o vuelve a reducirse, el peso de los servicios de la deuda sobre la escasa disponibilidad de divisas lleva a la necesidad de contraer nuevos préstamos internos, en una "espiral de endeudamiento"” (pág. 147).

El presente estudio tiene como objetivo principal analizar las variables de crecimiento económico en el Ecuador desde el periodo 2004 al 2015, cuya finalidad es contrastar las aristas de crecimiento versus el estudio realizado por Thirlwall en el largo plazo y determinar las verdaderas causales de desarrollo en el país.

Se proponen como objetivos específicos: a.) Analizar los componentes que conforman las exportaciones e importaciones anuales del Ecuador para el periodo 2004-2015; b.) Descomponer la balanza de pagos ecuatoriana para verificar el efecto crecimiento-inversión en el país; c.) Observar el comportamiento de los activos y pasivos financieros del país (periodo 2004-2015) y discutir si están variables han influenciado en el resultado económico anual.

\section{Metodología}

Para la elaboración de este trabajo se empleó el diseño retrospectivo que permitió la obtención de los datos y juicios correctos, estos contribuyeron a un desarrollo continuo de la relación entre los argumentos de Thirlwall y la realidad del país.

En el presente se analizó el comportamiento de las series que determinan el crecimiento económico como lo son el producto interno bruto y la evolución de la balanza de pagos mediante investigación bibliográfica. Los datos presentados se tabularon con periodicidad anual desde el año 2004 al 2015 y fueron recopilados de las diferentes instituciones públicas.

Dentro de los datos se hizo una separación de los bienes de naturaleza petrolera y no petrolera, la cual permite analizar el factor dependiente de la estabilidad económica del Ecuador. La fuente de estos datos proviene del Banco Central del Ecuador y el Banco Mundial.

Para alcanzar tales montos por ingresos en exportaciones petroleras fue importarte distinguir las variables que intervienen o influyen durante la operación con la OPEP u otras naciones que compran el crudo, estas fueron la cantidad de barriles que extraen las refinerías y el precio por barril de petróleo, tal variable como el precio es establecido por la OPEP de acuerdo a la volatilidad en el mercado.

En cuanto a las importaciones y exportaciones, estas se expresaron en miles de dólares, en medida corriente. En la cuenta de exportaciones, se halló la segregación $(+)$ Mercancías según la CAE, (+) Ajuste por comercio no registrado, (+) Reparación de bienes $(+)$ Bienes adquiridos en puerto por medios de transporte, las sumas de todas estas cuentas totalizaron los montos exportados en bienes tangibles (en millones de dólares).

En cuanto a las exportaciones no petroleras, estas fueron clasificadas en tradicionales y no tradicionales. Las exportaciones tradicionales fueron aquellos bienes que se exportaron con mayor frecuencia, la mayoría de los bienes en esta lista de caracterizan por ser materias primas o productos con bajo valor agregado (primarios), según la segregación que se encontró en el informe de la balanza comercial tenemos: banano y plátano, camarón, café y elaborados, cacao, atún y pescado, flores, etc.

Entre las exportaciones no tradicionales que comercian el Ecuador se exceptúan a los tradicionales y toman mayor protagonismo los manufacturados que son resultado de un proceso productivo. Entre ellos se pueden encontrar prendas o accesorios de vestir, artesanías, extractos vegetales, plásticos, caucho, etc.

La tasa de crecimiento del PIB estuvo definida de manera porcentual de acuerdo a estudios realizados por la CEPAL. Estos datos fueron utilizados como base para realizar un análisis descriptivo mediante histogramas con líneas de tendencia, lo cual fue contrastado teóricamente con el apartado de restricción de la balanza de pagos de la Ley de Thirlwall.

De esta manera, se realizó una explicación de cómo el comportamiento de los componentes del crecimiento ecuatoriano tuvo compatibilidad con la estabilidad de la balanza de pagos o mejor llamado comercio internacional, la cual señaló Thirlwall, puede llegar a ser insostenible si el déficit de la balanza de pagos incrementa, dando finalmente como resultado una contracción de la demanda y la pérdida de competitividad con los recursos actuales.

La balanza de pagos es considerada como un balance en donde se contabilizan todos los movimientos o transacciones entre el Ecuador y el resto del mundo. La balanza de pagos ecuatoriana al menos consta de tres partes importantes: cuenta corriente, cuenta de capital y financiera, y cuenta para contabilizar los errores y omisiones. Las cifras registradas son similares a los asientos contables en donde se debe cumplir el principio de partida doble.

Parte del análisis de la cuenta corriente se realizó cuando se revisaron las importa- 
ciones en relación a las exportaciones y se determinaron los superávits/déficits comerciales, y en términos generales se observaba que para los últimos años desde el 2010 teníamos saldos negativos en la balanza comercial.

Para este efecto, aquellas cuentas de la balanza de pagos que tengan saldo deudor poseerán el signo (+), mientras que aquellas cuentas que tengan un saldo acreedor tendrán el signo $(-)$, el saldo neto que se encuentre pude bifurcarse en un superávit o déficit. Para que exista un equilibrio entre el total de los débitos y créditos es necesario ajustar el saldo final en un financiamiento para que se cumpla la igualdad.

Cabe recalcar que las cuentas pertenecientes a "otras inversiones" son consideradas como cuentas de pasivo, por lo que la naturaleza siempre será en el haber (acreedoras), si se encuentran saldos deudores (-) en una cuenta de pasivo significa pagos netamente devengados de una deuda total o pagos anticipados al prestamista.

En cuanto a los pasivos para la balanza de pagos 2015 se obtuvo información del Banco Mundial para justificar que los préstamos externos tienen una influencia importante (un $94,76 \%$ de participación sobre el total de los pasivos financieros). Para el período 2014-2015 el país consiguió importantes créditos de instituciones internacionales como el
Deutsche Bank, Banco Mundial, China Exim Bank y el Fondo Interamericano de Reservas, la mayoría de estos créditos tenían como justificativo el financiamiento de proyectos de carreteras e hidroeléctricas, financiamiento del déficit en la cuenta corriente y la inyección de capital en las diferentes infraestructuras públicas ubicadas a nivel nacional.

A través de gráficos estadísticos, se observó el comportamiento anual de las variables económicas de crecimiento y su relación, cuyos ciclos podrían explicar y dar evidencia de las diferentes etapas en el dinamismo de la economía del Ecuador y si estos factores son aplicables según Thirlwall.

Resultados

\section{Evolución de las exportaciones en el Ecuador}

El total de las exportaciones nos permite deslumbrar el desempeño en los sectores productivos de todo el país, de hecho, es la medida más común empleada para medir la producción anual. El comportamiento de este indicador en Ecuador indica un claro proceso de transición hacia una etapa de competitividad en el mercado internacional.

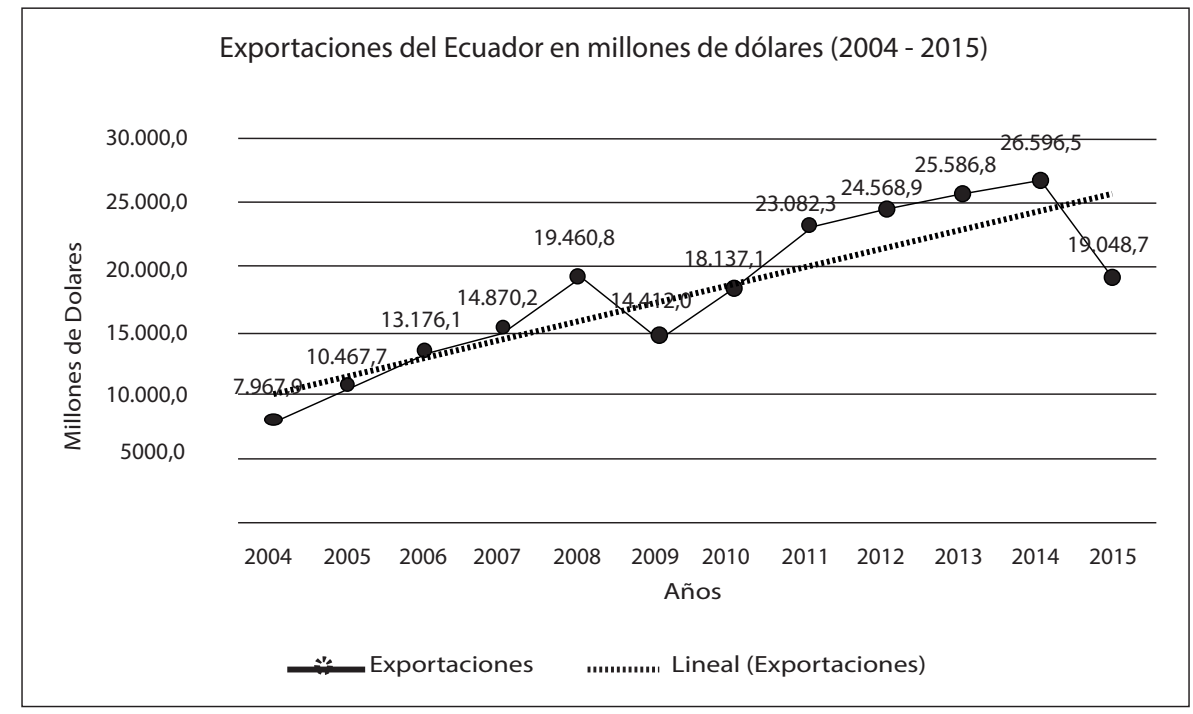

Figura 1. Exportaciones del Ecuador en millones de dólares (2004-2015). Fuente: Banco Central del Ecuador.

En el período entre 2004 y 2015 se observan exportaciones con tendencia creciente, alcanzando su valor más alto en el 2014 (\$26.596,50 millones de dólares) y sufriendo un crecimiento decreciente en el 2009 ( $\$ 14.412,0$ millones de dólares), el cual es considerado el monto más bajo en el período de análisis debido a la gran depresión del 2009 durante la crisis financiera internacional en EEUU. No obstante, en 2015 se registra una nueva caída en las exportaciones (ahora ubicadas en \$19.048,75 millones de dólares). La tasa promedio de cre- cimiento para el período 2004 al 2015 es del $5.50 \%$, confirmando así el crecimiento sostenible que ha mantenido el país, y que tiene un peso importante en el cálculo del PIB anual.

Del total de las exportaciones que realiza el país realizaremos una segregación más: las petroleras y las no petroleras, de esta manera podremos comprobar la sensibilidad y la dependencia del crudo. Entre las exportaciones petroleras tenemos que estas se clasifican a su vez en petróleo crudo (WTI) y derivados (gasolina, GLP, nafta, etc.). 


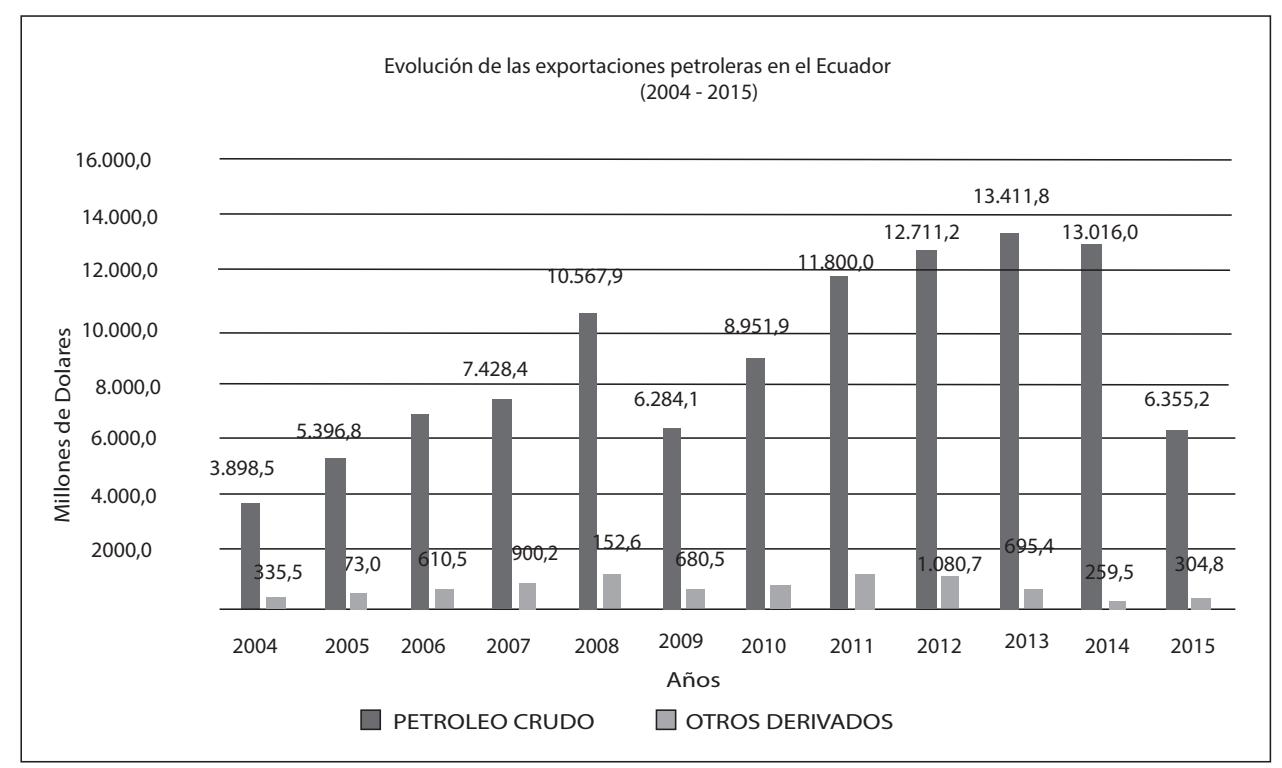

Figura 2. Evolución de las exportaciones petroleras del Ecuador en millones de dólares (2004-2015). Fuente: Banco Central del Ecuador.

Como se observa en el gráfico de barras es predominante la exportación del crudo sobre los derivados petroleros, de hecho, al ponderar dichos valores sobre el total de las exportaciones petroleras se obtiene que el crudo está por encima del 90\% del total (promedio anual del período 2004 al $2015=92.74 \%$ ), mientras que los derivados tienen un peso máximo del 10\% (promedio anual del período 2004 al $2015=7.26 \%$ ).

Finalmente, el peso promedio que tiene el total de las exportaciones petroleras sobre el total de mercancías exportadas según la CAE (Corporación Aduanera Ecuatoriana) es de 52.96\%.

\section{Evolución de las importaciones en el Ecuador}

Las importaciones pueden considerarse potencialmente positivas o negativas en el modelo de crecimiento de una economía, dependiendo en la medida que se les permita sustituir a los bienes de consumo nacional. No obstante, importar mesuradamente también puede lograr la eficiencia de las empresas nacionales, siendo un factor indiscutible para la competitividad y el crecimiento. En Ecuador, los montos de importaciones han tendido a incrementarse, siendo las materias primas uno de los principales artículos comprados por el sector secundario.

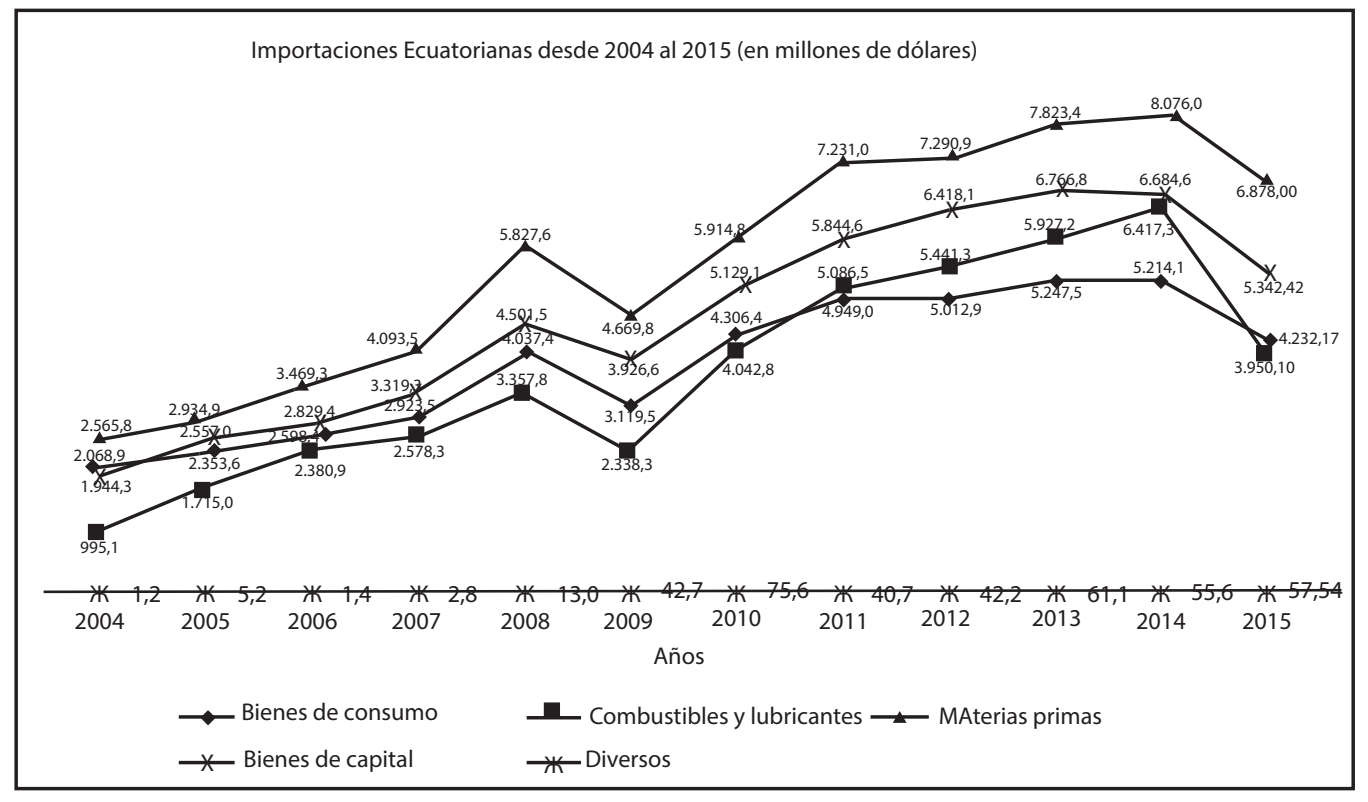

Figura 3. Importaciones Ecuatorianas desde 2004 al 2015 (en millones de dólares). Fuente: Banco Central del Ecuador. 
Las principales importaciones que realiza el país tienen que ver con: bienes de consumo, combustibles y lubricantes, materias primas, bienes de capital (inmuebles) y otros bienes diversos. Se observa que las importaciones de materias primas empleadas, para producir nacionalmente, están por encima de las demás, en segundo lugar se encuentran las compras bienes inmuebles productivos (bienes de capital). Desde el año 2011 se observa que las importaciones en combustibles y lubricantes ( $\$ 5.086,5$ millones) superan a los bienes en consumo (\$4.949,o millones), en el 2015 se restablece el comportamiento desde 2004 hasta 2010 de ambas cuentas, las importaciones en bienes de consumo se colocan nuevamente por encima de los bienes importados en combustibles y lubricantes (\$4.232,17 millones sobre $\$ 3.950,10$ millones).

Una comparativa entre las exportaciones e importaciones ecuatorianas revela que la tendencia y el comportamiento son similares. En el período del 2004 al 2015 se observa que el intervalo (2004-2009) las importaciones son menores a las exportaciones, para estos años por definición se tiene una balanza comercial positiva (superávit comercial) para el $2004(\$ 284), 2005(\$ 758,3), 2006$ $(\$ 1.768,4), 2007(\$ 1.823,0), 2008(\$ 1.548,7)$ y $2009(\$ 143,6)$ expresado en millones de dólares.

\section{Evolución de la balanza de pagos en el Ecuador}

La balanza de pagos es considerada como un balance en donde se contabilizan todos los movimientos o transacciones entre el Ecuador y el resto del mundo. La balanza de pagos ecuatoriana al menos consta de tres partes importantes: cuenta corriente, cuenta de capital y financiera, y cuenta para contabilizar los errores y omisiones.

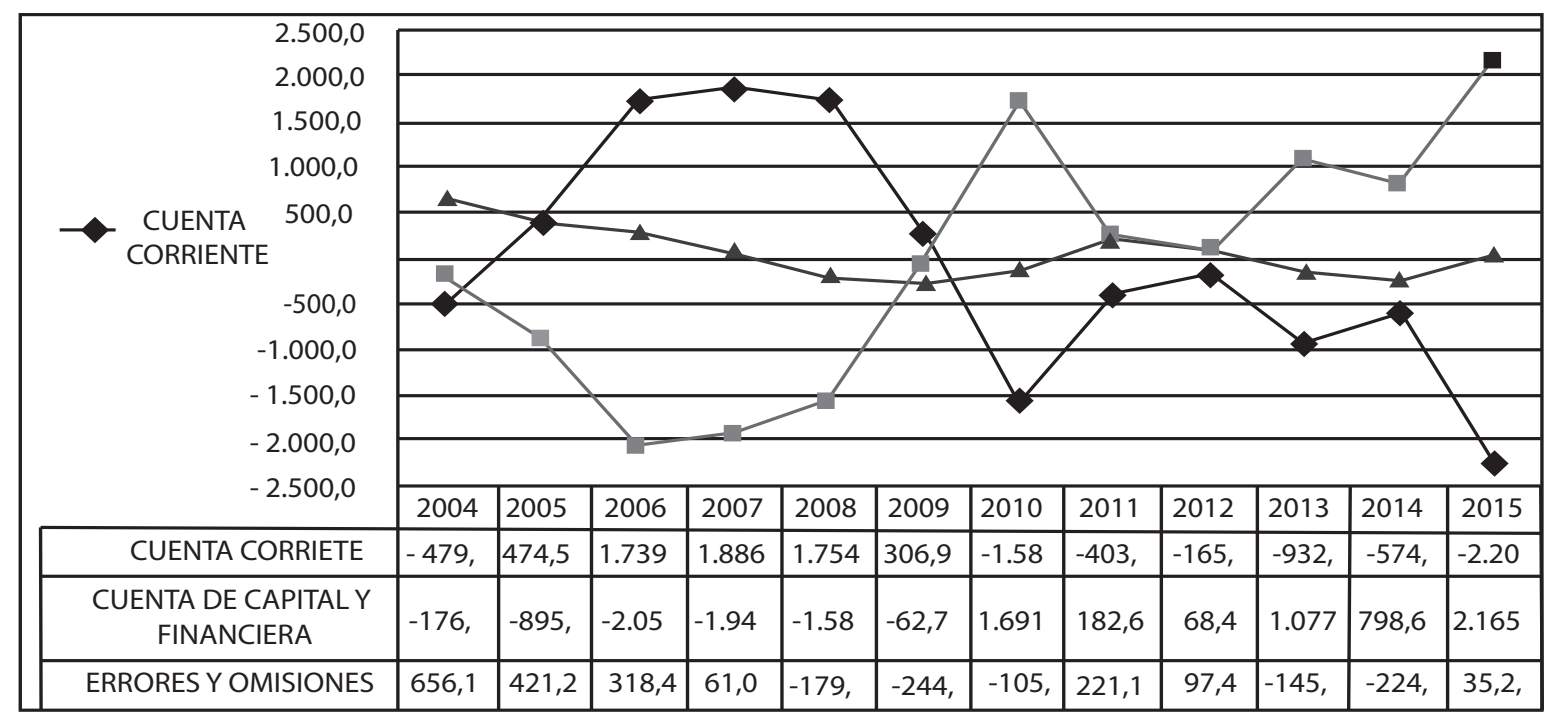

Figura 4. Comportamiento de la balanza de pagos ecuatoriana en millones de dólares (2004 al 2014). Fuente: Banco Central del Ecuador.

El comportamiento de la balanza de pagos en el Ecuador se ha caracterizado por elevados valores de deuda encontrados en la cuenta capital y financiera. Si totalizamos los montos desde el 2004 al 2015 tenemos los siguientes rubros: Cuenta corriente acumulada (- \$170,38 millones), cuenta de capital y financiera acumulada (- \$740,93 millones), cuenta emisiones y errores ( $\$ 911,31$ millones).

En la cuenta principal de capital y financiera tenemos que el mayor peso se encuentra sobre los movimientos financieros que realiza el país, dentro del mismo tenemos las inversiones nacionales y extranjeras, al igual que los créditos (activos) y préstamos (pasivos) que contrae el país. Para 2015 la carga porcentual de los movimientos financieros fue igual al $102.34 \%$ del total de la cuenta capital y financiera. Las distribuciones siguientes en la cuenta financiera para el año 2015 se encontraron de la siguiente manera: \$1.321,42 millones para la inversión directa que contempla la inversión nacional y la extranjera, $\$ 1.473,38$ que contempla la inversión en cartera de activos y pasivos, $-\$ 2.113,18$ distribuidos en créditos, préstamos, monedas/depósitos, y otros pasivos, finalmente se tienen activos de reserva equivalentes a $\$ 1.453,11$. 
Tabla 1Análisis de distribuciones porcentuales en la cuenta 2.2.3 "Otra inversión" (Cifras en millones de dólares)

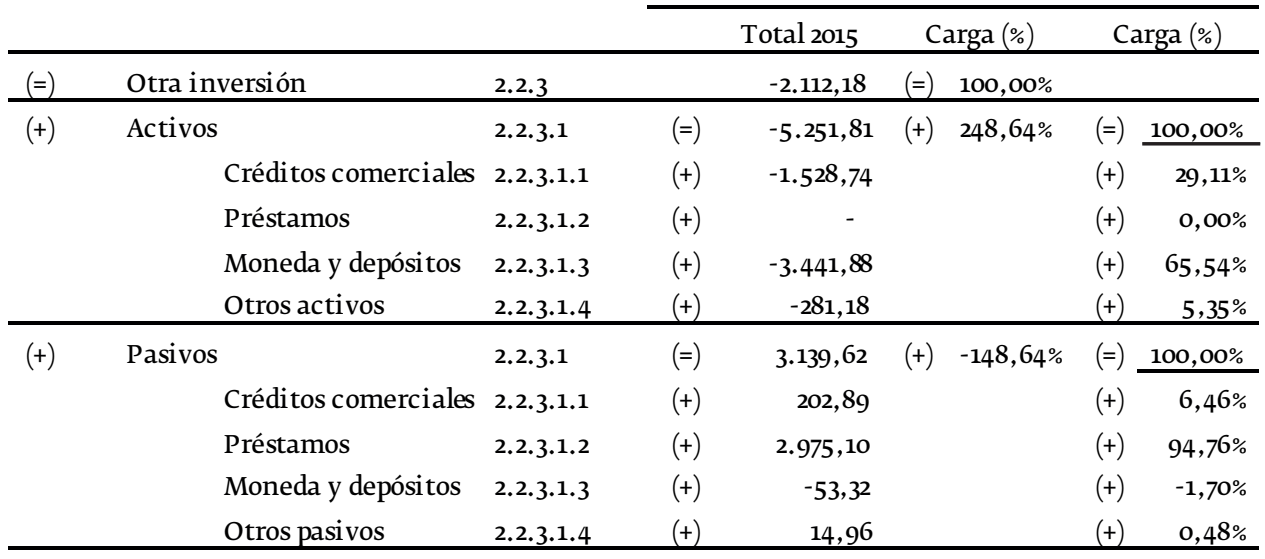

De las subcuentas mencionadas en el párrafo anterior analizaremos la cuenta 2.2.3 denominada "otra inversión", la cual cerró en la balanza de pagos 2015 con un saldo de - $\$ 2.112,18$ millones de dólares. Esta cuenta se subdivide en activos y pasivos, los registros realizados en estas cuentas corresponden a inversiones contraídas con entes no residentes y que no son considerados como una inversión directa.

Entre los activos financieros a los que el estado tiene derecho a cobro tenemos un peso del $248,64 \%$ y en total suman $-\$ 5.251,81$ millones de dólares con naturaleza deudora (mayor en el debe que el haber), en cuanto a las obligaciones de pago que el estado tiene con terceros nos encontramos con $-148,64 \%$ y en total suman \$3.139,62 millones de dólares con natura- leza acreedora (mayor en el haber que el debe).

En la cuenta del activo para la balanza de pagos 2015 se puede observar que la cuenta más relevante es la de moneda y depósitos, mientras que el pasivo más representativo lo constituyen los préstamos estatales.

El peso de las monedas y depósitos en la economía representa $65,54 \%$ del total de los activos financieros de la economía, seguido de los créditos comerciales otorgados por el activo representan desembolsos del $29,11 \%$. Cabe mencionar que, bajo economías dolarizadas, un cambio en las divisas (dadas por devaluaciones) afectará directamente a la balanza de pagos en un aumento del precio de las importaciones, haciendo más atrayente la producción nacional para el consumo.

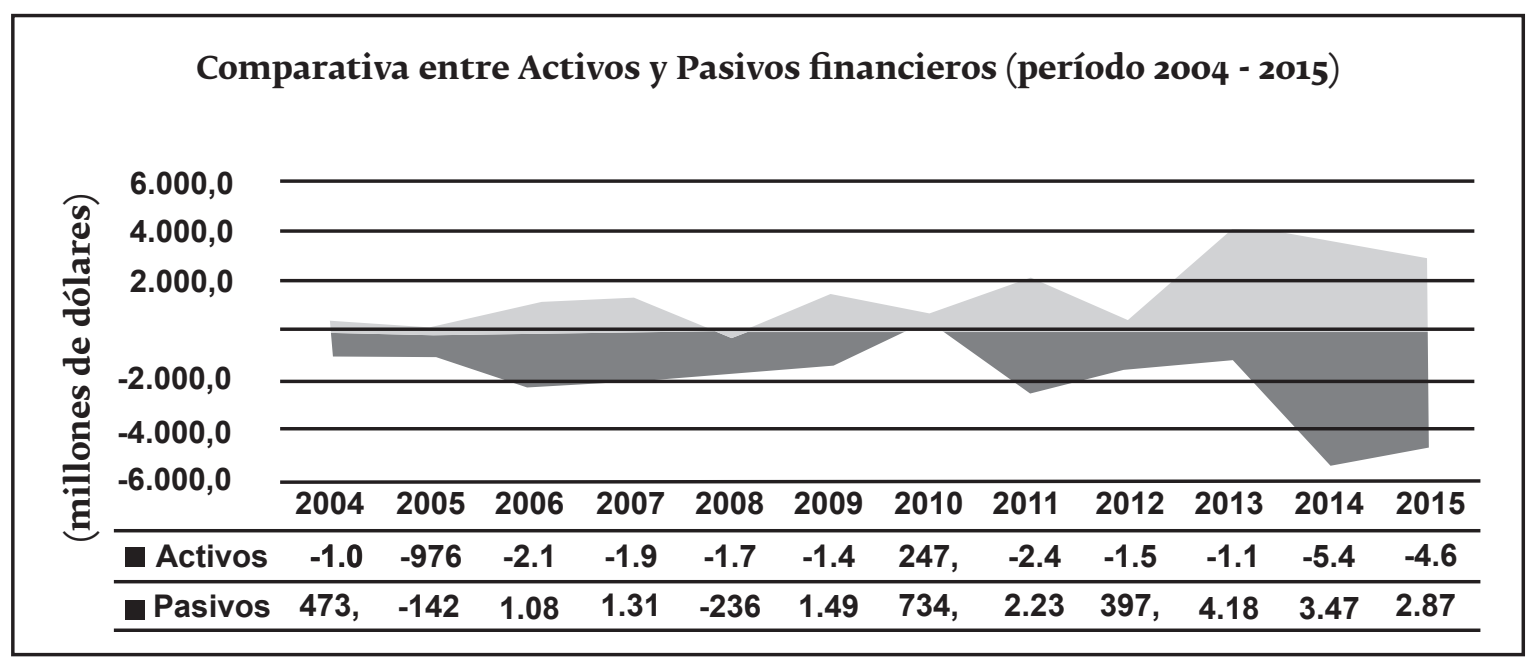

Figura 5. Comparativa entre Activos y Pasivos financieros (período 2004 al 2014).

Fuente: Banco Central del Ecuador. 
Una comparativa entre los activos y pasivos financieros del país nos permite observar una preeminencia de los pasivos financieros sobre los activos. En donde, los mayores desembolsos para los activos se presentan para el 2014 en $-\$ 5.453,8$ millones de dólares y la mayor deuda adquirida se presenta en el 2013 con \$4.158,2 millones de dólares.

Según la Organización para la Cooperación y el Desarrollo Económico (2008):”Las deudas dentro y entre diferentes niveles de gobierno están consolidadas; un préstamo de un nivel de gobierno a otro representa un activo y un pasivo equivalente para el gobierno como un todo y se cancela (está "consolidado") para el sector del gobierno general" (pág. 208).

Por tales razones, es permisible que los saldos ubicados en las cuentas de la balanza de pagos estén disponibles apenas el país emita deudas, documentos relacionados con recursos financieros, ya que estas existencias deben estar sujetas a revisión, ya que el deterioro de la transparencia en esta clase de documentos oficiales es muy común con el fin de mantener una calma momentánea de los economistas críticos que vigilan de forma macro al país.

\section{Discusión}

Para países dolarizados, es importante evaluar la liquidez de las cuentas externas del país porque estos no cuentan con emisión monetaria y al ocasionarse cambios sustanciales en la economía, se pueden tomar medidas correctas para contrarrestar aquellos comportamientos económicos dañinos.

Según Ocegueda (2006), refiriéndose al estudio de Thirlwall, "se encuentra un vínculo entre las tasas de crecimiento observadas y la razón existente entre las tasas de crecimiento de las exportaciones y la elasticidad del ingreso de la demanda por importaciones $\chi^{\varepsilon_{-}} \mathrm{m}$ " (pág. 50).

Aquellas situaciones represivas en la economía, llevan a la reflexión del costo que tienen las tasas de interés, lo cual desestimula las inversiones brutas de capital fijo, al no haber esta acumulación, hay un alto costo para la sociedad que no se puede compensar.

Según este enfoque hay una bifurcación en la balanza de pagos para que un país pueda crecer bajo una política específica:

1. Para superar los desfases de la balanza de pagos, el gobierno planifica a través de una política de expansión en la demanda; el reto es alcanzar la tasa de crecimiento potencial, ya que en caso de no hacerlo se recortará la expansión de la demanda desmotivando las inversiones y las expansiones en tecnología.

Según Fernández, y otros (2006) "en los últimos treinta años, las tasas de paro se han situado en niveles muy altos y de for- ma persistente (...) lo que hace imposible considerarlo como paro cíclico susceptible de ser eliminado aplicando políticas de expansión de la demanda" (pág. 23).

2. Una economía tiene una balanza de pagos equilibrada y logra expandir la demanda por encima del nivel óptimo de producción en el corto plazo. En el largo plazo la elevación de la demanda por encima de los niveles de trabajo normales, se la podría explotar más mediante la compra de activos de capital, lo que desencadena en un aumento de la producción empleando las mismas cantidades de capital y trabajo, lo que permite un crecimiento en óptimas condiciones. Sin duda las economías especializadas son aquellas que consiguen crecer por encima de la productividad. En su obra Mungaray, Palacio, \& Ruiz (2002) reflexionan que estas economías "experimentan mayor crecimiento de la productividad, pero (...) que éste se ve superado por el incremento de la demanda, son las que crean más y mejor empleo. La mayor productividad permite pagar salarios más elevados sin detrimento de los beneficios" (pág. 53).

Así, la competitividad está condicionada bajo la posibilidad de los rendimientos que arrojan la estructura de producción y la manera en expandir la demanda.

Ambas bifurcaciones concluyen en una importante restricción del crecimiento y de la igualdad en la balanza de pagos, para el primer caso se tendrán déficits que se necesitan financiar, y en la otra cara de la moneda, al maximizar la demanda se estimulará la oferta en el mercado de empleos y la presión fomenta a que los niveles de precio se incrementen.

Por ejemplo, en el caso de Ecuador, la presencia de déficits se ha hecho presente debido al crecimiento de pasivos en la balanza de pagos 2015. Se puedo observar que los préstamos externos tienen un $94,76 \%$ de participación sobre el total de los pasivos financieros.

Así, para el período 2014-2015 el país consiguió importantes créditos de instituciones internacionales como el Deutsche Bank, Banco Mundial, China Exim Bank y el Fondo Interamericano de Reservas, la mayoría de estos créditos tenían como justificativo el financiamiento de proyectos de carreteras e hidroeléctricas, financiamiento del déficit en la cuenta corriente y la inyección de capital en las diferentes infraestructuras públicas ubicadas a nivel nacional.

\section{Conclusión}

La dinámica que propone Thirlwall formula el crecimiento desde un punto de vista enfocado en la demanda y el libre intercambio externo (la demanda agregada, exportaciones e impor- 
taciones). Hemos hecho un repaso de las variables involucradas en el crecimiento del país, de cuyas valoraciones se obtienen cifras optimistas y al mismo tiempo, cifras que preocupan porque arrojan resultados insatisfactorios.

Adicional el modelo de Thirlwall sugiere que existe una restricción en la balanza de pagos relacionado con un lento crecimiento de las tasas en las exportaciones, las cuales desde el 2004 al 2015 se ha presentado con tendencia al incremento (tasa promedio ponderada de 10,39\%).

En cuanto a las importaciones, se observó que estas también continúan creciendo, pero a diferencia de las exportaciones, no parecen mostrar una señal de debilitamiento. De tal manera tenemos que la tasa promedio de crecimiento de las importaciones durante estos últimos 11 años es de $11,18 \%$, revelando de a poco una relevancia en la elasticidad de las compras superiores con respecto a las ventas al extranjero.

El comportamiento de las tasas en aumento en las exportaciones e importaciones y las delicadas variaciones en la balanza de pagos, se conjugan para demostrar que los eventos ocurridos, justificados con las cifras presentadas, nos intenten explicar cómo los principios de esta ley aún sobreviven en el Ecuador, explicando de esta manera el porqué de los ascensos y descensos en las tasas de crecimiento. Por ahora, los datos muestran que en Ecuador juegan un papel importante aumentar la exportación nacional para expandir su crecimiento.

Con respecto a la evolución que ha tenido la balanza de pagos, los montos de la cuenta financiera tienen una mayor participación en la estructura de la balanza de pagos, durante los últimos 11 años solamente la totalidad de la cuenta capital y financiera representan importantes desembolsos efectuados por motivo de deuda contraída en donde intervienen los recursos financieros. Tomando en cuenta que, para países dolarizados, es importante evaluar la liquidez de las cuentas externas del país porque estos no cuentan con emisión monetaria y al ocasionarse cambios sustanciales en la economía, se pueden tomar medidas correctas para contrarrestar aquellos comportamientos económicos dañinos.

Contrastando la literatura de Thirlwall, concluimos que esta disparidad entre importaciones y exportaciones indica que, aunque el país tenga una balanza comercial negativa, al mismo tiempo han existido restricciones que han limitado las exportaciones; pues una segunda posibilidad sería que el país haya sufrido un proceso de desindustrialización, la cual no es convincente. Para cuál sea el caso, podríamos afirmar que la pérdida de competitividad se ha hecho presente.

\section{Referencias}

Alvarado, R., \& Iglesias, S. (2017). Sector externo, restricciones y crecimiento económico en Ecuador. Revista Problemas del Desarrollo, 191(48), 83-106.

Arestis, P., McCombie, J., \&Vickerman, R. (2006). Growthand Economic Development: Essays in Honour of A. P. Thirlwall. Northampton: MPG Books Ltd, Bodmin, Conwall.

Banco Central del Ecuador. (2007). Cambios en la compilación de la balanza de pagos. Retrieved from Moneda y depósitos en dolarización: http://contenido.bce.fin.ec/documentos/Estadisticas/SectorExterno/BalanzaPagos/MetodoCambioComBP.pdf

BancoCentraldelEcuador.(n.d.). Estadísticas. Retrieved from Balanza de pagos analítica (período 2002-2015 IIT): http:// contenido.bce.fin.ec/documentos/Estadisticas/SectorExterno/BalanzaPagos/boletin52/2_BdPAnalitica.xls

Banco Central del Ecuador. (n.d.). Estadísticas. Retrieved from Balanza de pagos en bienes (período: 2002-2015 IIT) : http://contenido.bce.fin.ec/documentos/Estadisticas/ SectorExterno/BalanzaPagos/boletin52/5_Bienes.xls

Banco Mundial. (n.d.). Databank. Retrieved from Crecimiento del PIB (\% anual): http://datos. bancomundial. org/indicador/NY.GDP. MKTP.KD.ZG/countries/ec-xj?display=graph

Bértola, L. (200o). Ensayos de historia económica: Uruguay en la región y el mundo. Montevideo: Ediciones TRILCE.

Bresser-Pereira, L., Oreiro, J., \& Marconi, N. (2015). Developmental Macroeconomics: New Developmentalism as a Growth Strategy. New York: Routledge, Taylor \& Francis Group. CEPAL. (2006). El estructuralismo latinoamericano, México: Siglo XXI Editores, S.A.

CEPAL. (n.d.). CEPALSTAT. Retrieved from Base de datos: http://interwp.cepal.org/sisgen/ ConsultaIntegrada. as p? Id A plicacion $=6$ \& $i-$ d Te ma $=131 \&$ id Indicador $=2204 \&$ id i o $\mathrm{ma}=\mathrm{e}$

Clavijo, P., \& Ros, J. (2015). La Ley de Thirlwall: una lectura crítica. Investigación Económica, 74(300), 11-40.

Davidson, P. (2015, Noviembre 04). A Post Keynesian Positive Contribution to "Theory". Retrieved from Taylor \& Francis Online: https://www.tandfonline.com/doi/abs/10.1080/o1603477.1990.11489849?j o u r n a l c o d e $=m$ p k e 20

De Gregorio, J. (2007). Macroeconomía: Teorías y Políticas (Primera ed.). México: Pearson Educación.

Fernández, J., Vallés, J., Ogando, O., Pedrosa, R., Miranda, B., Gómez, J., \& Urueña, B. (2006). Principios de política económica (Primera ed.). Madrid: Delta, Publicaciones Universitarias.

Ibarra, C. (2015). A "La Ley de Thirlwall: Una Lectura crítica" de Pedro Clavijo Y Jaime Ros. Investigación económica, 74(292), 41-45.

King, J. E. (2015). Advanced Introduction to Post Keynesian Economics. Cheltenham: Elgar Advanced Introductions.

Landa, H. (2017). Crecimiento, competitividad y restricción externa en América Latina. Investigación Económica, 76(300), 53-80.

Lavoie, M. (2014). Post-Keynesian Economics: New Foundations. Cheltenham: Edwars Elgar Publishing Limited.

León, M. (2016). Diagnóstico y perspectiva de la economía ecuatoriana en 2016. Friedrich Ebert Stiftung (FES-ILDIS) Ecuador, 1-28.

López, J., \& Cruz, A. (2015, Noviembre 03). "Thirlwall's Law” and Beyond: The Latin American Experience. Retrieved from Taylor \& Francis Online: https://www.tandfonline.com/doi/abs/10.1080/01603477.2000.11490253?$\mathrm{j}$ o $\mathrm{u}$ r n a l C o d e $=m$ p k e 2 o

Morones, A. (2016). Crecimiento económico en México: restricción por la balanza de pagos. Ensayos Revista de Economía, 35(1), 39-58.

Ocegueda, J. (2006). La restricción externa al crecimiento económico de México: El impacto de las reformas estructurales. México: Universidad Autónoma de Baja California.

Ortiz, C. (2013). Economic growth consequences of structural stagnation: A two-sector model of productive diversification. Medellín: Universidad 
de Antioquía, Facultad de Ciencias Económicas. Parkín, M. (2004). Economía (Sexta ed.). México: Pearson Educación Rivadeneira, R., \& Buitrón, C. (2017). Panorama económico de la deuda externa e interna del Ecuador periodo 2000-2016. Revista Publicando, 4(10), 431-447. Rodríguez, G., Loor, H. , \& Navas, W. (2019). Casode estudio: e default del Ecuador 2008-2009. Ciencia Digital, 3(1), 1-14. Sala-I-Martin, X. (2000). Apuntes de creciminto económico (Segunda ed.). Barcelona: Antoni Bosch, Editor, S.A. Soukiazis, E., \& Cerqueira, P. (2012). Models of Balance of
Payments Constrained Growth: History, Theory and Empirical Evidence. Basingstoke: PALGRAVE MACMILLAN. Thirlwall, A., \& Pacheco, P. (2017). Economics of development: Theory and Evidence (Décima ed.). London: Red Globe Press.

Vanoni, G., \& Rodríguez, C. (2017). Estrategias de crecimiento implementadas por los grupos económicos (2007-2016). Revista INNOVAR Journal, 27(64), 39-54. 